\title{
Evolutionary oligopoly games with cooperative and aggressive behaviors
}

\author{
Gian Italo Bischi ${ }^{1} \cdot$ Fabio Lamantia ${ }^{2,3}$ (D)
}

Received: 10 April 2020 / Accepted: 15 August 2020 / Published online: 4 September 2020

(c) The Author(s) 2020, corrected publication 2020

\begin{abstract}
We propose an oligopoly model where players can choose between two kinds of behaviors, denoted as cooperative and aggressive, respectively. Each cooperative agent chooses the quantity to produce in order to maximize her own profit as well as the profits of other agents (at least partially), whereas an aggressive player decides the quantity to produce by maximizing his own profit while damaging (at least partially) competitors' profits. At each discrete time, players face a binary choice to select the kind of behavior to adopt, according to a proportional imitation rule, expressed by a replicator equation based on a comparison between accumulated profits. This means that the behavioral decisions are driven by an evolutionary process where fitness is measured in terms of current profits as well as a weighted sum of past gains. The model proposed is expressed by a nonlinear two-dimensional iterated map, whose asymptotic behavior describes the long-run population distribution of cooperative and aggressive agents. We show under which conditions one of the following long-run behaviors prevails: (i) all players choose the same strategy; (ii) both behaviors coexist according to a mixed stationary equilibrium; and (iii) a self-sustained (i.e. endogenous) oscillatory (periodic or chaotic) time pattern occurs. The influence of memory and that of the levels of cooperative/aggressive attitudes on the dynamics are analyzed as well.
\end{abstract}

Keywords Oligopoly games · Partial cooperation $\cdot$ Replicator dynamics · Discrete dynamical systems $\cdot$ Stability $\cdot$ Bifurcations

Fabio Lamantia

fabio.lamantia@unical.it

Gian Italo Bischi

gian.bischi@uniurb.it

1 Department of Economics, Society, Politics (DESP), University of Urbino, Urbino, Italy

2 Department of Economics, Statistics and Finance (DESF), University of Calabria, Rende, Italy

3 Faculty of Economics, VŠB Technical University of Ostrava, Ostrava, Czech Republic 


\section{Introduction}

In classical oligopoly models, the goal of each firm is to maximize (or at least to increase) its own profits. Typically in these models, strategic interaction only occurs indirectly because of the influence of competitors' decisions on economic quantities regarding other competitors, such as the market price through the demand function or the production costs through cost externalities [for example, in the presence of spillover effects, see, e.g., D’Aspremont and Jacquemin (1988)].

However, even direct strategic interaction may be considered if some firms in the oligopoly market include (at least partially) competitors' profits in their own objective function they wish to maximize (or increase). Following this idea, Cyert and DeGroot (1973) introduce partial cooperation by inserting a fraction of competitors' profit (modulated by a coefficient of cooperation) in each objective function (see also Bischi et al. 2010, chapter 4). This may be related to the fact that firms wish to produce inside industrial districts where similar firms operate, in order to take advantage of common infrastructures, knowledge spillovers, cross-shareholdings, etc. On the contrary, different situations may lead some firms to increase their own profits while trying to decrease (at least partially) competitors' ones. This attitude, which may be denoted as aggressive, may be justified in industrial competition contexts in which it is preferred to weaken the opponent even at the expense of obtaining a lower profit for themselves: a position in a ranking can be achieved both by increasing own gains and/or by decreasing competitors' ones, an attitude that has been denoted as spiteful behavior in the literature [see, e.g., Vriend (2000); Vallée and Yildizoglu (2009)]. The literature on strategic delegation also fits into this context, see Fershtman and Judd (1987) and De Giovanni and Lamantia (2016).

The issue of the evolution of cooperation in prisoner dilemma games has been widely discussed. An important point of view to explain possible behaviors different from those found with the maximization of expected utility concerns the distinction between material payoffs and utility payoffs, see Ahn et al. (2001) and Andreoni and Miller (2002). Ahn et al. (2001) also stress the different results in terms of evolution of cooperation when players are matched randomly or repeatedly with the same agent, thus introducing the idea of a kind of memory behind the behavior. Agents can adopt forms of "social preferences," sacrificing part of their payoffs to increase the average payoffs of the population, as explained in Charness and Rabin (2002). Furthermore, when it comes to situations of strategic interaction even with two agents but which can be chosen within a larger population, the literature refers to the concept of "indirect reciprocity" to explain the evolution of cooperative behavior that would otherwise hardly emerge, see Leimar and Hammerstein (2001) and Nowak and Sigmund (2005) for an extensive overview on the point.

Janssen (2008) proposes a model in which agents play one-shot prisoner's dilemma games with the option of withdrawal. The trustworthiness of others when withdrawal is not chosen is assessed through the expected utility of cooperative and defective behavior with the logit model. Janssen (2008) also differentiates between the agents' utility and expected payoff. The analysis is carried out with artificial agents and shows that the presence of learning about the reliability of opponents increases the general level of cooperation. 
In this paper, we propose an oligopoly model where firms may behave sometimes as partial cooperators and sometimes as aggressive agents. Firms can switch from a kind of behavior to another one through an evolutionary model driven by a replicator equation where fitness is measured in terms of accumulated profits (monetary payoffs) and actions are guided by extended utility functions encouraging cooperative or aggressive behavior (utility payoffs). This setup is similar to the classic Hawk-Dove game in ecology, like the one proposed by Smith (1982) as a prototypical evolutionary game, just at the very beginning of this research field. As it is well known, a HawkDove one-shot game can give rise to a classical prisoner dilemma, where Hawk (or aggressive) behavior is dominant with respect to Dove (or cooperative) one. Hommes et al. (2018) is a relevant reference for the modeling of evolutionary oligopolies with different behavioral rules.

Given that in contexts of industrial competition collusive behaviors are generally not allowed, we assume that cooperative behavior is not determined by an agreement between the agents but by the willingness of each company to go in the direction of a less strong competition that still helps the whole industry to make higher profits. In some sense, the various behaviors are basically implemented to introduce forms of indirect reciprocity in the market. For this reason, each firm chooses at the pre-commitment level which strategy to adopt (cooperative or aggressive) and then observes the choice of its opponent to determine the quantities to be produced and obtains the consequent profit. For the same reason, we do not introduce "punishments to defectors" that in many cases can indeed steer the system toward cooperation but that are out of the picture in the context at hand.

Our contribution, moreover, unlike many other similar works, considers accumulated profit as a measure of fitness instead of current profit only. For this reason, we explore the role of memory on the dynamics of the model, or of history, both with analytical methods and with numerical explorations, usually necessary when dealing with global dynamics of nonlinear maps.

We completely characterize the case here proposed, which is the linear oligopoly setup with linear inclusion of one's opponent's profit in the extended objective function. Here, we present the analytical results that apply to all possible levels of cooperative or aggressive behavior. We manage, in this simple context, to characterize the model for each possible value of these parameters.

In this way, we add some insights to the vast literature on the evolutionary stability of the Walrasian equilibrium (or more generally to aggressive behavior) in an oligopolistic market, which originated with Vega-Redondo (1997), see Alós-Ferrer (2004), Vallée and Yildizoglu (2009), Apesteguia et al. (2010) and Radi (2017) and references therein. The evolutionary oligopoly model here proposed shows that the Walrasian equilibrium, that we retrieve when firms are "aggressive," is an equilibrium of the model but it is not always an evolutionary stable one.

In particular, we show the presence of a subspace of parameters in which no pure strategy (cooperative or aggressive behavior) dominates so that the dynamics do not converge to a boundary equilibrium with the presence of only the monomorphic configuration of the population. This, in particular, occurs when the cooperative behavior consists of incorporating only a small portion of the profit of the adversaries while the aggressive behavior consists in the heavy penalty toward the adversary. In this 
case, the presence of an equilibrium of coexistence of the two strategies (polymorphic configuration of the population) is demonstrated. However, this equilibrium can be unstable and gives rise to complex dynamics. We show in particular how these dynamics are influenced by the parameters related to the level of cooperation and by the amount of memory, which is stabilizing in our case. In this regard, it is interesting to note that the role of memory in the literature is not always univocal: sometimes it is locally stabilizing or destabilizing [see Hommes et al. (2012)], sometimes irrelevant for local stability properties of equilibria but influencing the global dynamics of the system (see Bischi et al. (2015) and Bischi et al. (2020)). For instance, Bischi et al. (2018) find that a short memory is destabilizing compared to the case without memory but a long memory (at the uniform limit) is highly stabilizing.

All results about existence of equilibria, their stability and bifurcations are reported in the paper also in the dynamic extension with memory. Through global analysis tools, we also explore, numerically, cases of coexistence of attractors. Furthermore, we present at the end of the paper what are the possible dynamic trends for the total profit of the industry when the main parameters of the model vary.

The paper is organized as follows. Section 2 presents the basic setup of the model with production strategy choices and evolutionary switches of behaviors based on exponential replicator dynamics with memory, as well as the particular formulation of the model in a market characterized by linear demand and cost functions with firms adopting Nash play to make their production choices. Section 3 collects the main analytical results concerning existence, stability and local bifurcations of the equilibrium points of the model. In Sect. 4, some numerical results are described to investigate bifurcation diagrams, coexistence of attractors each with its own basin of attraction and the dynamics of total industry profits. Finally, the last section is devoted to some concluding remarks and possible extensions of the dynamic model.

\section{The model}

Let us consider an oligopoly market with $N$ ex ante identical firms that produce homogeneous goods. At each discrete time $t$, a couple of firms, say firm $i$ and firm $j$, is assumed to be randomly selected among the firms of the population to engage a duopoly game and each of them can choose between two different (and in some sense contrasting) kinds of behavior: the first one, denoted as cooperative behavior, means that player $i$ adopting it decides quantity $q_{i}$ to maximize

$$
S_{i}=\pi_{i}\left(q_{i}, q_{j}\right)+\theta \pi_{j}\left(q_{j}, q_{i}\right)
$$

where $\pi_{i}\left(q_{i}, q_{j}\right)$ is player's $i$ expected payoff, which depends on the choice by player $j$ as well, and parameter $\theta \in[0,1]$ measures the amount (or intensity) of cooperative behavior; the second one, denoted as aggressive behavior, means that player $i$ decides quantity $q_{i}$ to maximize

$$
V_{i}=\pi_{i}\left(q_{i}, q_{j}\right)+\rho \pi_{j}\left(q_{j}, q_{i}\right)
$$


with $\rho \in[-1,0]$, where $|\rho|$ measures the amount (or intensity) of aggressive behavior.

To select quantities, firms can use an "optimal" (or at least satisfying, i.e. suboptimal, or adaptive) rule in order to get maximum (or increasing) payoff. Such behavioral rules may depend on the computational abilities of a firm or on the available information set. Anyway, we assume that each firm uses any of such rules to decide its own next period output. For instance, let us focus on the ones commonly proposed in the specialized literature such as Nash rule, best reply, gradient rule or local monopolistic approximation rule, etc. (see, e.g., Bischi et al. (2010) and references therein for an overview on these rules). Let us denote by $q_{s}(t)$ and $q_{v}(t)$ the quantities chosen at time $t$ according to the cooperative and aggressive objective functions, respectively, in (1) and (2). If firm $i$ chooses cooperative behavior, then it sets $q_{s}(t+1)$ to maximize (or at least increase) objective $S_{i}$ in (1) and expects to earn at time $t+1$ the following payoff:

$$
\pi_{i}^{e}\left(q_{s}(t+1), q_{-i}(t+1)\right)
$$

for a given production $q_{-i}(t+1)$ set by firm $i$ 's competitor. Similarly, if firm $i$ chooses aggressive behavior, it sets $q_{v}(t+1)$ to maximize (or at least increase) objective $V_{i}$ in (2) and has expected profits

$$
\pi_{i}^{e}\left(q_{v}(t+1), q_{-i}(t+1)\right) .
$$

Firms are assumed to behave cooperatively or aggressively according to an evolutionary switching mechanism where fitness is identified with profits. Let us consider a population of $N$ firms, where the current share of cooperators is $r=r(t)=\frac{N_{c}(t)}{N}$, $N_{c}(t)$ being the number of cooperators at time $t$ in the population. Of course, $r(t) \in[0,1]$ and the number of aggressive firms is given by the complementary fraction $N_{a}(t)=N(1-r(t))$. As usual in the evolutionary game approach, the share $r(t)$ approximates the probability Pr of extracting a firm with cooperative behavior in a random sampling and $1-r(t)$ is the probability of meeting an aggressive firm. So, the average profit at time $t$ of a firm with cooperative behavior is given by

$$
\bar{\pi}_{s}^{*}(t)=r(t) \pi_{s s}^{*}+(1-r(t)) \pi_{s v}^{*}
$$

where $\pi_{s s}^{*}$ represents the profit gained by a firm with cooperative behavior engaged in a duopoly contest with another firm adopting the same behavior, whereas $\pi_{s v}^{*}$ represents the profit obtained by a firm with cooperative behavior when its opponent adopts aggressive behavior.

Analogously, the average profit of a firm with aggressive behavior is

$$
\bar{\pi}_{v}^{*}(t)=r(t) \pi_{v s}^{*}+(1-r(t)) \pi_{v v}^{*}
$$

where $\pi_{v s}^{*}$ is the profit gained by an aggressive firm against one with cooperative behavior and $\pi_{v v}^{*}$ represents the profit obtained by each firm in a duopoly contest involving two aggressive firms. We assume an (exponential) replicator equation [in 
the form proposed by Cabrales and Sobel (1992), see also Hofbauer and Sigmund (2003)] to simulate the time evolution of $r(t)$

$$
r(t+1)=\frac{r(t) e^{\gamma \bar{\pi}_{s}^{*}(t)}}{r(t) e^{\gamma \bar{\pi}_{s}^{*}(t)}+(1-r(t)) e^{\gamma \bar{\pi}_{v}^{*}(t)}}=\frac{r(t)}{r(t)+(1-r(t)) e^{-\gamma \mathbb{F}(t)}}
$$

where $\gamma \geq 0$ is the intensity of choice and $\mathbb{F}(t)=\bar{\pi}_{s}^{*}(t)-\bar{\pi}_{v}^{*}(t)$ represents the fitness gain (i.e., competitive advantage) of cooperators with respect to aggressive agents.

Notice that quantities are set by considering "augmented" objective functions $S$ or $V$ in (1) and (2), whereas the profit (or payoff) associated with each behavior is obtained by considering expected accrued own profits, where the augmented term is omitted. This is in line with the so-called indirect evolutionary approach, described by Königstein and Müller (2000) and, more recently, by Alger and Weibull (2013) [see also Kopel et al. (2014) and Kopel and Lamantia (2018)] for an application to dynamic oligopolies). According to this approach, the evolution of behaviors, such as aggressive or cooperative attitudes in our model, depends on an "objective" measure of fitness, such as firms' profits, whereas the choice of strategies, given by production decisions in our case, depends on a "subjective" utility such as the augmented functions $S$ or $V$ here proposed.

Another key ingredient that we consider in the model is the presence of memory. As explained below, we are interested in considering what happens when agents embed into the fitness measure also the performance of past strategies. The details on this point are given below.

\subsection{Linear setup}

Let us consider the duopoly contest with linear demand $p=a-b\left(q_{i}+q_{j}\right)$, with $a>0, b>0$, and linear costs $C(q)=c q, c>0$, to develop the easiest case and study how the model looks like. The cases with more complicated demand and cost functions [such as with isoelastic demand, see, e.g., Puu (1991)], or nonlinear cost functions as well as cost functions with externalities due to spillovers [see, e.g., Bischi and Lamantia (2002) and Bischi et al. (2015)] can be then dealt following a similar way. In the linear setup, firm's $i$ profit is given by

$$
\pi_{i}=(p-c) q_{i}
$$

and under cooperative behavior firm $i$ selects quantity $q_{i}$ in order to maximize

$$
\begin{aligned}
S_{i} & =\pi_{i}\left(q_{i}, q_{j}\right)+\theta \pi_{j}\left(q_{j}, q_{i}\right) \\
& =(p-c) q_{i}+\theta(p-c) q_{j},
\end{aligned}
$$

whereas under aggressive behavior firm $i$ sets quantity to maximize

$$
\begin{aligned}
V_{i} & =\pi_{i}\left(q_{i}, q_{j}\right)+\rho \pi_{j}\left(q_{j}, q_{i}\right) \\
& =(p-c) q_{i}+\rho(p-c) q_{j}
\end{aligned}
$$




\subsubsection{Equilibrium under best reply: Nash play}

Let us assume that each firm follows a best reply strategy to decide the next period production. ${ }^{1}$ Under cooperative behavior, firm's $i$ best reply is

$$
q_{s}\left(q^{e}\right)=\frac{a-c-b q^{e}(1+\theta)}{2 b}
$$

which is easily obtainable by solving the F.O.C. $\frac{\partial S_{i}\left(q_{i}, q^{e}\right)}{\partial q_{i}}=0$ with respect to $q_{i}$.

If both firms are cooperators, then the Nash Equilibrium is given by the intersection of best reply reaction curves, where both firms produce the same quantities:

$$
q_{s s}^{*}=\frac{a-c}{b(3+\theta)}>0
$$

where the usual condition $a>c$ is assumed. In this case, both firms earn

$$
\pi_{s s}^{*}=\frac{(a-c)^{2}(1+\theta)}{b(3+\theta)^{2}} .
$$

It is plain that in the case $\theta=0$ (no cooperation) the usual Cournot-Nash duopoly equilibrium is obtained, whereas in the other limiting case $\theta=1$ (total cooperation) the monopoly solution is got, as both firms share the same objective of maximizing the joint profit.

Analogously, if both firms choose aggressive behavior, similar results follow by substituting $\theta$ with $\rho$. Thus, best reply under aggressive behavior of both players is given by

$$
q_{v}\left(q^{e}\right)=\frac{a-c-b q^{e}(1+\rho)}{2 b}
$$

and Nash Equilibrium is given by production plans

$$
q_{v v}^{*}=\frac{a-c}{b(3+\rho)}>0
$$

with profits

$$
\pi_{v v}^{*}=\frac{(a-c)^{2}(1+\rho)}{b(3+\rho)^{2}}
$$

It is trivial to observe that for $\rho=0$ the equilibrium reduces to the standard Cournot-Nash one, whereas in the other limiting case $\rho=-1$ the Walrasian equilibrium is obtained, which is characterized by zero profits to the firms. Finally, in the

1 Cases of different kinds of strategies for production decisions, involving different degrees of information and computational ability (such as gradient rules, LMA, etc.) can be considered as well, especially in situations with nonlinear demand or cost functions). 
mixed case the Nash Equilibrium is given by

$$
q_{s v}^{*}=\frac{(a-c)(1-\theta)}{b(3-\theta-\rho-\theta \rho)}>0 ; \quad q_{v s}^{*}=\frac{(a-c)(1-\rho)}{b(3-\theta-\rho-\theta \rho)}>0
$$

Notice that these equilibria are always nonnegative since $a>c$ has been assumed and the following condition always holds under our parameter setting: $3-\theta-\rho-\theta \rho>0$. In the following, we will always assume that $\theta$ and $\rho$ are not both equal to zero; otherwise, the distinction between the two behaviors vanishes.

The corresponding profits at equilibrium (11) are given by

$$
\pi_{s v}^{*}=\frac{(a-c)^{2}(1-\theta \rho)(1-\theta)}{b(3-\theta-\rho-\theta \rho)^{2}}
$$

and

$$
\pi_{v s}^{*}=\frac{(a-c)^{2}(1-\theta \rho)(1-\rho)}{b(3-\theta-\rho-\theta \rho)^{2}}
$$

where $\pi_{s v}^{*}$ represents the profit at equilibrium for the player that chooses $q_{s v}^{*}$ when its opponent chooses $q_{v s}^{*}$.

In the following, we focus on Nash play, see Hommes et al. (2018), which occurs when each agent chooses the quantities to be produced for its chosen behavior based on the expressions found in (7), (9) and (11). In this case, it is easy to show that prices are always strictly positive in all configurations of the game for each pair of parameters $\theta \in[0,1]$ and $\rho \in[-1,0]$.

This basic example shows the prisoner's dilemma structure of the game at hand, as it is

$$
\pi_{s s}^{*}>\pi_{v v}^{*}
$$

so both firms are better off if they both choose to cooperate, but being

$$
\pi_{s v}^{*}<\pi_{v s}^{*}
$$

a firm that chooses cooperative behavior is worst off if the competitor chooses aggressive behavior.

In addition, in the linear setup, the following inequalities hold (see "Appendix")

$$
\pi_{s v}^{*}<\pi_{s s}^{*}<\pi_{v s}^{*}
$$

which shows that if agent $i$ cooperates, its payoff is always greater in the case that also agent $j$ chooses to cooperate; however, if agent $j$ cooperates, agent $i$ is better off by not cooperating.

This situation is quite common in Hawk-Dove games [see, e.g., Smith (1982)]. As it is well known, even if in a one-shot Hawk-Dove game that assumes the form of a prisoner dilemma the aggressive behavior is dominant, in the case of a repeated game, as assumed in an evolutionary setting, cooperative behavior may emerge in the long run. So, in the following we shall consider the model with replicator dynamics (5) 
under the assumption of linear demand and cost functions with firms following Nash play, as outlined above. In this case, the fitness gain of cooperators with respect to aggressive agents, denoted as $\mathbb{F}(t)$ in (5), becomes

$$
\begin{aligned}
\mathbb{F}(t) & =\bar{\pi}_{s}^{*}(t)-\bar{\pi}_{v}^{*}(t) \\
& =r(t) \pi_{s s}^{*}+(1-r(t)) \pi_{s v}^{*}-\left[r(t) \pi_{v s}^{*}+(1-r(t)) \pi_{v v}^{*}\right] \\
& =\alpha r(t)+\beta
\end{aligned}
$$

with

$$
\begin{aligned}
& \alpha=\pi_{s s}^{*}+\pi_{v v}^{*}-\pi_{s v}^{*}-\pi_{v s}^{*} \\
& \beta=\pi_{s v}^{*}-\pi_{v v}^{*}
\end{aligned}
$$

It is possible to show that $\alpha<0$, whereas $\beta$ can take any sign in the considered parameter space (see "Appendix").

Summing up, by the inequality $\pi_{s s}^{*}<\pi_{v s}^{*}$ in (16), the cooperative strategy is never dominant: when player $j$ plays cooperatively, agent $i$ is better off by playing aggressively. On the other hand, playing aggressively can be a dominant strategy. This occurs when $\pi_{s v}^{*}<\pi_{v v}^{*}$, that is when $\beta<0$ : playing aggressively is more rewarding to agent $i$ both when agent $j$ plays cooperatively $\left(\pi_{s s}^{*}<\pi_{v s}^{*}\right)$ and when agent $j$ plays aggressively $\left(\pi_{s v}^{*}<\pi_{v v}^{*}\right.$ ) [recall that condition $\pi_{s s}^{*}<\pi_{v s}^{*}$ always holds]. As a consequence, when $\beta<0, \mathbb{F}(t)=\bar{\pi}_{s}^{*}(t)-\bar{\pi}_{v}^{*}(t)<0$ for any share of cooperators $r(t) \in[0,1]$, so that any dynamic adjustment based on fitness should punish the use of the cooperative strategy.

On the other hand, more interesting situations arise when $\beta>0$ : as in this case both conditions $\pi_{s v}^{*}>\pi_{v v}^{*}$ and $\pi_{v s}^{*}>\pi_{s s}^{*}$ hold, then for any agent the best choice is to play differently from the competitor. As we show below, this means that in the population of firms the long-run distribution of behaviors can converge to a polymorphic configuration with coexistence of both behaviors as there is no dominance of one pure strategy over the other.

The case $\beta<0$ seems to confirm what is already known in the literature, i.e., that the Walrasian equilibrium is evolutionarily stable, by adding that not only the Walrasian equilibrium is evolutionarily stable, but also any "milder" aggressive behavior is so. In addition, the case $\beta>0$ leads to something new that adds to the literature and to the debate on the evolutionary stability of an equilibrium different from the Walrasian one and, more generally, on the instability of the aggressive behavior in an oligopoly market, see Vega-Redondo (1997), Radi (2017), Alós-Ferrer (2004), Apesteguia et al. (2010), Schaffer (1989) and Cerboni-Baiardi and Naimzada (2018).

However, the stability of an equilibrium when $\beta>0$ depends on the other behavioral parameters like the intensity of choice $\gamma$ in the evolutionary process and on the amount of past profits memory, which is now introduced in the model. 


\subsection{The model with memory}

As it is well known, in an economic context the replicator equation expresses a proportional imitation rule, where strategies of firms getting higher profits are imitated with a probability that is proportional to their expected payoff, see Hofbauer and Sigmund (1998). In the following, we shall assume that such imitation rule is not only based on a comparison of current profits but also of accumulated profits. In other words, in addition to considering last period profits only, the fitness measure takes into account past strategies performances as well, referred to simply as memory in the following. Memory is embedded in the fitness through a weighted average of past profit values, with weights expressing a profit smoothing factor. We introduce in the model such a "fitness with memory" by proposing the following recursive (or inductive) definition of time $t$ accumulated profits with exponential smoothing:

$$
\begin{aligned}
y(t) & =(1-\omega)\left(\bar{\pi}_{s}^{*}(t)-\bar{\pi}_{v}^{*}(t)\right)+\omega y(t-1)= \\
& =(1-\omega) \mathbb{F}(t)+\omega y(t-1)= \\
& =(1-\omega)[\alpha r(t)+\beta]+\omega y(t-1)
\end{aligned}
$$

where the weight $\omega \in[0,1)$ is a memory parameter that states how much of past profits contribute to current fitness. When $\omega=0$ the no-memory case is obtained, whereas in the opposite limiting case $\omega \rightarrow 1^{-}$the current expected gain $\mathbb{F}(t)=\bar{\pi}_{S}^{*}(t)-\bar{\pi}_{v}^{*}(t)$ is neglected and relative fitness is constant.

All in all, the dynamic model we shall consider below is obtained by the iteration of the following two-dimensional map $T: A \rightarrow A$, where $A=[0,1] \times(-\infty,+\infty)$ :

$$
T:\left\{\begin{aligned}
r(t+1)= & \frac{r(t)}{r(t)+(1-r(t)) e^{-\gamma y(t)}} \\
y(t+1)= & (1-\omega)[\alpha r(t+1)+\beta]+\omega y(t) \\
& =(1-\omega)\left[\frac{\alpha r(t)}{r(t)+(1-r(t)) e^{-\gamma y(t)}}+\beta\right]+\omega y(t)
\end{aligned}\right.
$$

Summing up, map (19) depends on the following parameters:

$-\omega \in[0,1)$ (amount of memory);

$-\gamma \in[0,+\infty)$ (intensity of choice);

$-a \in(0,+\infty)$ (maximum selling price) and $b \in(0,+\infty)$ (opposite of demand slope);

$-c \in[0,+\infty)$ (marginal cost);

$-\theta \in[0,1]$ (amount of cooperative behavior);

$-\rho \in[-1,0]$ (opposite of the amount of aggressive behavior).

With the exception of the amount of memory $\omega$ and the intensity of choice $\gamma$, all other parameters of map (19) are subsumed in the aggregate parameters $\alpha \in(-\infty, 0)$ and $\beta \in \mathbb{R}$. 


\section{Equilibrium points, local stability and bifurcations}

In this section, analytical results about existence and local stability of equilibrium points of the discrete dynamical system (19) are provided. Moreover, based on local linearization, a study of the local bifurcations is presented in order to characterize stability losses and qualitative changes of the dynamical properties of each equilibrium as the parameters of the model vary. However, as usual in nonlinear dynamic models, this study is not sufficient to give a complete characterization of the long-run dynamic properties of the system, so a global analysis based on numerical and geometric methods will be performed in the next section in order to discover the existence of attracting sets that are more complex than stationary equilibria, as well as to study their basins of attraction in the presence of multistability, i.e., when several coexisting attracting sets are present.

The first proposition concerns the existence of equilibrium points.

Proposition 1 (Equilibrium points) The dynamical system (19) always admits the following two boundary equilibrium points:

$$
E_{0}=(0, \beta) \text { and } E_{1}=(1, \alpha+\beta)
$$

and a third inner equilibrium given by

$$
E_{*}=\left(-\frac{\beta}{\alpha}, 0\right)
$$

provided that the aggregate parameter $\beta>0$ is given. In terms of amount of aggressive and cooperative behavior $\rho$ and $\theta, \beta>0$ is equivalent to the following conditions:

$$
-1 \leq \rho<3-2 \sqrt{3} \text { and } 0 \leq \theta<\frac{-\rho^{2}+6 \rho+3}{3 \rho^{2}+6 \rho-1}
$$

Proof The dynamic variable $r$ is stationary for (19) when $r(t+1)=r(t)$, and this occurs for $r=0$ or $r=1$ or $y=0$. From these three conditions, the three equilibrium points are calculated from the algebraic system obtained from (19) with $r(t+1)=$ $r(t)=r$ and $y(t+1)=y(t)=y$. Notice that at an equilibrium $y(t)=y(t-1)=y^{*}$ and, from (18), it follows that $y^{*}=(1-\omega) \mathbb{F}(t)+\omega y^{*}$; hence, $y^{*}=0$ implies $\mathbb{F}=0$ so that it holds that $\bar{\pi}_{s}^{*}(t)=\bar{\pi}_{v}^{*}(t)$. Equilibrium $E_{*}$ is feasible provided that $-1 \leq \frac{\beta}{\alpha} \leq 0$. From the negativity of $\alpha$ (see "Appendix") and from (16), the inequality $\alpha+\beta<0$ follows so that condition $-1 \leq \frac{\beta}{\alpha}$ is always satisfied. Thus, the condition for the feasibility of $E_{*}$ reduces to $\frac{\beta}{\alpha} \leq 0$, i.e., $\beta \geq 0$. The conditions for the positivity of $\beta$ are obtained in "Appendix."

Equilibrium $E_{0}$ represents a homogeneous situation with no cooperators (i.e., all aggressive agents), whereas $E_{1}$ represents a homogeneous situation with all cooperators (i.e., no aggressive agents). Equilibrium $E_{*}$ is characterized by identical profits 
gained by cooperative and aggressive players being $\bar{\pi}_{s}^{*}(t)=\bar{\pi}_{v}^{*}(t)$. This occurs whenever $\beta>0$, i.e., when $\pi_{s v}^{*}>\pi_{v v}^{*}$. From an economic point of view, the existence of such a mixed equilibrium occurs whenever equilibrium profits are such that, given an aggressive opponent, a firm is better off by playing nonaggressively. This can occur only in situations in which aggressive play heavily punishes competitors (low $\rho$ ) and cooperators do not add much of the competitor's profits into their objective function (low $\theta$ ), as specified in the parameter region (20). At $E_{*}$, the distribution of both strategies lies in the interior of the interval $(0,1)$. Thus, the dynamics converge to a heterogeneous (or mixed) population of aggressive/cooperative agents if $\beta>0$, with the prevalence of cooperators if $0<\beta<-\frac{\alpha}{2}$ and the prevalence of aggressive agents if $-\frac{\alpha}{2}<\beta<-\alpha$. This indifference situation at the mixed equilibrium is a standard occurrence in evolutionary games. The stability properties of these equilibrium points, as well as the related local bifurcations, are described by the following proposition.

\section{Proposition 2 (Stability Analysis)}

- The boundary equilibrium $E_{0}$ of the map (19) is a stable node if $\beta<0$ and a saddle point if $\beta>0$, with stable set along the vertical invariant edge $r=0$ and unstable set transverse to it.

- The boundary equilibrium $E_{1}$ is always a saddle point, with stable set along the vertical invariant edge $r=1$ and unstable set transverse to it.

- The interior equilibrium $E_{*}$ is stable if

$$
2 \alpha(1+\omega)-(1-\omega) \beta \gamma(\alpha+\beta)<0
$$

- At $\beta=0$ a transcritical bifurcation occurs at which $E_{*}=E_{0}=(0,0)$, and the two equilibria exchange their stability along the transverse invariant set.

- If $E_{*}$ is a feasible equilibrium, that is, conditions (20) are verified, then it loses stability through a flip bifurcation when the left-hand side of the expression in (21) becomes positive.

Proof In order to study the stability of the three equilibrium points, we follow the usual linearization procedure based on the study of the eigenvalues of the Jacobian matrix of (19)

$$
J(r, y)=\left[\begin{array}{cc}
\frac{e^{-\gamma y}}{\left(r+(1-r) e^{-\gamma y}\right)^{2}} & \frac{\gamma r(1-r) e^{-\gamma y}}{\left(r+(1-r) e^{-\gamma y}\right)^{2}} \\
\frac{\alpha(1-\omega) e^{-\gamma y}}{\left(r+(1-r) e^{-\gamma y}\right)^{2}} & \frac{\alpha \gamma(1-\omega) r(1-r) e^{-\gamma y}}{\left(r+(1-r) e^{-\gamma y}\right)^{2}}+\omega
\end{array}\right]
$$

computed at the equilibrium points. At $E_{0}$, we have

$$
J\left(E_{0}\right)=\left[\begin{array}{cc}
e^{\gamma \beta} & 0 \\
\alpha(1-\omega) e^{\gamma \beta} & \omega
\end{array}\right]
$$

a triangular matrix with eigenvalues $\lambda_{1}=e^{\gamma \beta}$, always positive and less than 1 if $\beta<0, \lambda_{2}=\omega \in[0,1)$. So, $E_{0}$ is a stable node if $\beta<0$ and a saddle point if $\beta>0$ 
with stable set along the vertical invariant edge $r=0$ and unstable set transverse to it. Analogously, at $E_{1}$, we have

$$
J\left(E_{1}\right)=\left[\begin{array}{cc}
e^{-\gamma(\alpha+\beta)} & 0 \\
\alpha(1-\omega) e^{-\gamma(\alpha+\beta)} & \omega
\end{array}\right]
$$

that is again a triangular matrix with eigenvalues $\lambda_{1}=e^{-\gamma(\alpha+\beta)}$, which is always greater than 1 being $\alpha+\beta<0$, and $\lambda_{2}=\omega \in[0,1)$. So, $E_{1}$ is a saddle point with stable set along the vertical invariant edge $r=1$ and unstable set transverse to it.

Finally, at the interior equilibrium $E_{*}$, the Jacobian matrix is given by

$$
J\left(E_{*}\right)=\left[\begin{array}{cc}
1 & -\frac{\beta \gamma(\alpha+\beta)}{\alpha^{2}} \\
\alpha(1-\omega) & -(1-\omega) \frac{\beta \gamma(\alpha+\beta)}{\alpha}+\omega
\end{array}\right]
$$

Thus, $E_{*}$ is locally asymptotically stable if the characteristic equation

$$
P(\lambda)=\lambda^{2}-\operatorname{Tr} \lambda+\operatorname{Det}=0
$$

where $\operatorname{Tr}$ and Det represent, respectively, the trace and the determinant of $J\left(E_{*}\right)$ :

$$
\begin{aligned}
\operatorname{Tr} & =1+\omega-(1-\omega) \frac{\beta \gamma(\alpha+\beta)}{\alpha} \\
D e t & =\omega,
\end{aligned}
$$

has roots with $|\lambda|<1$. Sufficient conditions for this are given by the Schur stability conditions [see, e.g., Gandolfo (2010), Medio and Lines (2001) and Elaydi (1995)]

$$
\left\{\begin{array}{l}
1-T r+\text { Det }=(1-\omega) \frac{\beta \gamma(\alpha+\beta)}{\alpha}>0 \\
1+\operatorname{Tr}+\text { Det }=2(1+\omega)-(1-\omega) \frac{\beta \gamma(\alpha+\beta)}{\alpha}>0 \\
\text { Det }=\omega<1
\end{array}\right.
$$

The third stability condition in (22) is clearly always satisfied. The first one in (22) is satisfied whenever $E_{*}$ is a feasible equilibrium, as feasibility of $E_{*}$, occurs provided that $\beta>0$, being $\alpha<0$ and $\alpha+\beta<0$. The second condition in (22) holds for

$$
2 \alpha(1+\omega)-(1-\omega) \beta \gamma(\alpha+\beta)<0
$$

and when it becomes positive, a real eigenvalue exits the unit circle through $\lambda=-1$, condition for the occurrence of a flip bifurcation. At $\beta=0$, a transcritical bifurcation occurs at which $E_{*}$ merges with $E_{0}, E_{*}=E_{0}=(0,0)$, and the two equilibria exchange their stability along the transverse invariant set (not the vertical one). Notice that differently from equilibrium $E_{0}$, there is no transcritical bifurcation at which $E_{*}=E_{1}=(1,0)$, and there is no stability exchange along the transverse invariant manifold as condition $\alpha+\beta=0$ is never satisfied. 
It is worth to notice that from (23) when $E_{*}$ is feasible, i.e., when $\beta>0$ so that $r=r_{*} \in(0,1)$, then $E_{*}$ is stable for $\gamma<\gamma_{F}$ where

$$
\gamma_{F}=\frac{2 \alpha}{\beta(\alpha+\beta)} \cdot \frac{1+\omega}{1-\omega}
$$

and $E_{*}$ undergoes a flip bifurcation at $\gamma=\gamma_{F}$. This shows the stabilizing role of the memory parameter $\omega \in[0,1)$ : in fact, ceteris paribus, the factor $\frac{1+\omega}{1-\omega}$ tends to plus infinity as $\omega \rightarrow 1^{-}$, thus enlarging the stability range of $E_{*}$.

Moreover, the stability condition (23) can be expressed in terms of the memory parameter $\omega: E_{*}$ is stable for $\omega>\omega_{F}$, where

$$
\omega_{F}=\frac{\gamma \beta(\alpha+\beta)-2 \alpha}{\gamma \beta(\alpha+\beta)+2 \alpha}
$$

whose denominator is always negative when $E_{*}$ is feasible. So, if also the numerator is negative, we have $0<\omega_{F}<1$, so that the mixed equilibrium $E_{*}$ is stable for high values of the memory parameter and it becomes unstable for decreasing memory.

An interesting condition is obtained by solving the same stability condition (23) with respect to the aggregate parameter $\beta$, because we get a second-degree inequality

$$
\gamma(\omega-1) \beta^{2}+\gamma \alpha(\omega-1) \beta+2 \alpha(\omega+1)<0
$$

If the discriminant of this second-degree polynomial is positive, then two positive solutions are obtained because of the Descartes' rule of signs (being the coefficients negative-positive-negative), say $0<\beta_{F 1}<\beta_{F 2}$, and the equilibrium $E_{*}$ is stable for $\beta<\beta_{F 1}$ or $\beta>\beta_{F 2}$. For example, in the case of no memory $\omega=0$, we get $\beta_{F 1,2}=-\frac{\alpha}{2} \pm \frac{\alpha}{2} \sqrt{1+\frac{2}{\gamma \alpha}}$.

The result that memory strengthens the stability of the coexistence equilibrium of aggressive and cooperative agents is in line with other results in the literature. For instance, Alós-Ferrer (2004) finds that the stability of the Walrasian equilibrium, demonstrated in Vega-Redondo (1997), is lost when memory is introduced into the system. Relatedly, Vriend (2000) shows that by introducing individual learning agents can abandon more aggressive strategies to move toward more cooperative solutions.

The stability results and bifurcation conditions here obtained will be used as a guide for some numerical explorations shown in the next section.

\section{Numerical explorations}

We now consider the dynamic model (19) and, given a fixed set of economic parameters $a, b, c$, we explore how the long-run evolution of population share between the two kinds of behavior is conditioned by the behavioral parameters $\theta, \rho$, as well as the evolutionary parameters $\gamma$ and $\omega$. Although the examples presented below are obtained with specific values assigned to the parameters, they are representative of the typical dynamics observed in the model. 

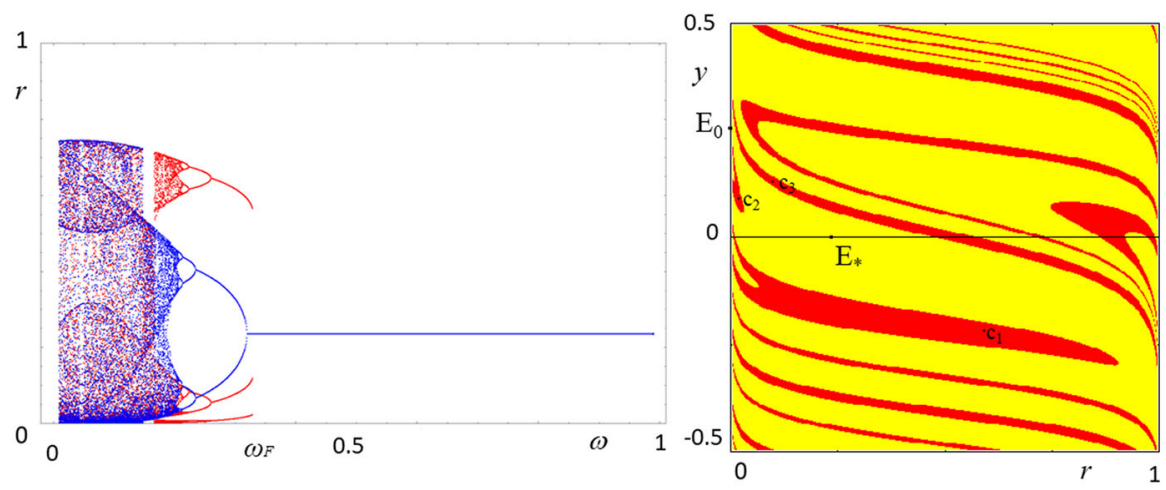

Fig. 1 Left: bifurcation diagram with parameters $a=4, b=0.5, c=0.5, \theta=0.25, \rho=-0.7, \gamma=20$ and taking the memory parameter $\omega \in[0,1)$ as bifurcation parameter; red points [blue points] represent the asymptotic portion of the trajectories starting from initial condition $(r(0), y(0))=(0.65,-0.03)$ $[(r(0), y(0))=(0.5,-0.01)]$. Right: basins of attraction of the stable equilibrium $E_{*}$ (yellow region) and of the coexisting stable cycle of period 3 with periodic points $\left(c_{1}, c_{2}, c_{3}\right)$ (red region) obtained for the same set of parameters as the bifurcation diagram in the left and with $\omega=0.32$ (color figure online)

A common practice to start a numerical investigation consists in the analysis of bifurcation diagrams, obtained by assigning increasing values to a given parameter (denoted as bifurcation parameter) whose values are reported in the horizontal axis of a Cartesian diagram, and representing a given number of asymptotic (or long-run) values in the corresponding vertical line, after a given transient portion of the trajectory has been neglected.

The bifurcation diagram obtained with the fixed set of parameters $a=4, b=0.5$, $c=0.5, \theta=0.25, \rho=-0.7$ and $\gamma=20$ and taking the memory parameter $\omega \in[0,1)$ as bifurcation parameter is represented in the left panel of Fig. 1. For this set of parameters, the values of the aggregate parameters $\alpha$ and $\beta$ can be computed, $\alpha \simeq$ -1.0784 and $\beta \simeq 0.2536$. As stated in Proposition 1 of Sect. 3 , the interior equilibrium $E_{*}$ exists, being $\beta>0$ and, by Proposition 2, it is stable for sufficiently high values of $\omega$, namely for $\omega>\omega_{F} \simeq 0.31$. When $\omega$ decreases until $\omega_{F}$, a flip bifurcation occurs at which the equilibrium loses stability and a stable cycle of period 2 is created. Then, as $\omega$ is further decreased, the usual period-doubling cascade occurs, leading to stable periodic cycles of increasing period, a classical route toward deterministic chaos. The densely covered part represents chaotic time patterns of the dynamic variables (as usual intermingled with periodic windows) along which high sensitivity with respect to small perturbations makes predictions quite difficult.

Additionally, the bifurcation diagram of Fig. 1 shows another form of uncertainty. In fact, in a range of the parameter $\omega$ around $(0.25,0.35)$, another attractor coexists, which can be reached for the same values of $\omega$ but starting from the different initial conditions. To emphasize its existence, we have plotted in the bifurcation diagram of Fig. 1, left panel, as red points all the trajectories of map (19) starting with initial condition $(r(0), y(0))=(0.65,-0.03)$ and as blue points all trajectories with initial condition $(r(0), y(0))=(0.5,-0.01)$. 
This coexistence of several attracting sets, each with its own basin of attraction, is also denoted as multistability [see, e.g., Bischi and Kopel (2003)]. In order to see how the phase plane $(r, y)$ of the dynamical system is shared by the different basins of attraction, and consequently to study how the long-run evolution of the system is determined by assigning different initial conditions (or exogenous perturbations that cause shifts of the initial state of the system) a phase portrait is shown in the right panel of Fig. 1, obtained with the same set of parameters as the bifurcation diagram with fixed $\omega=0.32$. In this picture, the yellow region represents the basin of the locally asymptotically stable equilibrium $E_{*}=(0.23,0)$, whereas the initial conditions taken in the red region lead to a periodic pattern of period 3 with periodic points $c_{1}=(0.59,-0.22), c_{2}=(0.018,0.09)$ and $c_{3}=(0.10,0.13)$. The particular structure of the basins expresses an important feature of nonlinear dynamic models with coexisting attractors, called sometimes "corridor stability," see, e.g., Leijonhufvud (1973) or Dohtani et al. (2007). This stream of literature stresses the fact that nonlinear dynamic models may have the property that small perturbations are recovered as far as they are confined inside the basin of attraction of a locally stable equilibrium, whereas larger perturbations lead to time evolutions that further depart from the equilibrium and go to the coexisting attractor in the long run. This remarkable phenomenon cannot be revealed by the propositions on local stability given in Sect. 3, based on the linear approximation of the dynamical system around the equilibrium points. The multistability and the related basins of attraction require a global dynamic analysis, often based on numerical and geometrical methods. If this numerical exploration is neglected, a study only based on local stability properties may even be misleading. On the other side, if the study is only limited to a numerical exploration, not guided by a previous analytical study of the dynamical system, then no useful conclusions can generally be obtained.

A similar study can be performed by taking the amount of cooperative behavior $\theta \in[0,1]$ as bifurcation parameter with the other parameters' values as in Fig. 1 and $\omega=0.3$, as shown in the left panel of Fig. 2. In this case, as the bifurcation parameter is increased, two flip bifurcations occur at the bifurcation values denoted as $\theta_{F_{1}} \approx 0.01721<\theta_{F_{2}} \approx 0.26783$, according to the second-degree bifurcation condition (24) discussed at the end of Sect. 3. In particular, the interior equilibrium where both behaviors coexist is stable for $0 \leq \theta<\theta_{F_{1}}$ and for $\theta>\theta_{F_{2}}$, whereas a stable oscillation of period 2 with periodic points around the unstable equilibrium $E_{*}$ characterizes the long-run dynamics between the two flip bifurcations. This implies that the role of an increasing attitude to partial cooperation, in the presence of strong aggressiveness of some firms due to the value of $\rho=-0.7$, is not univocal. Moreover, also in this case, the bifurcation diagram reveals that a range of the parameter exists where multistability occurs, as the stable cycle of period 2 coexists with a stable cycle of period 3, each attractor with its own basin of attraction (yellow and red points, respectively). The corresponding basins are represented in the right panel of Fig. 2, and again the complicated topological structure of the basins' boundaries is quite evident.

As the parameter of partial cooperation $\theta$ is further increased, the aggregate parameter $\beta$ decreases and the equilibrium $E_{*}$ is characterized by a smaller and smaller fraction $r(t)$ of cooperators, until $\beta=0$ when $\widehat{\theta} \approx 0.4531$ (see (20)): at this point, 

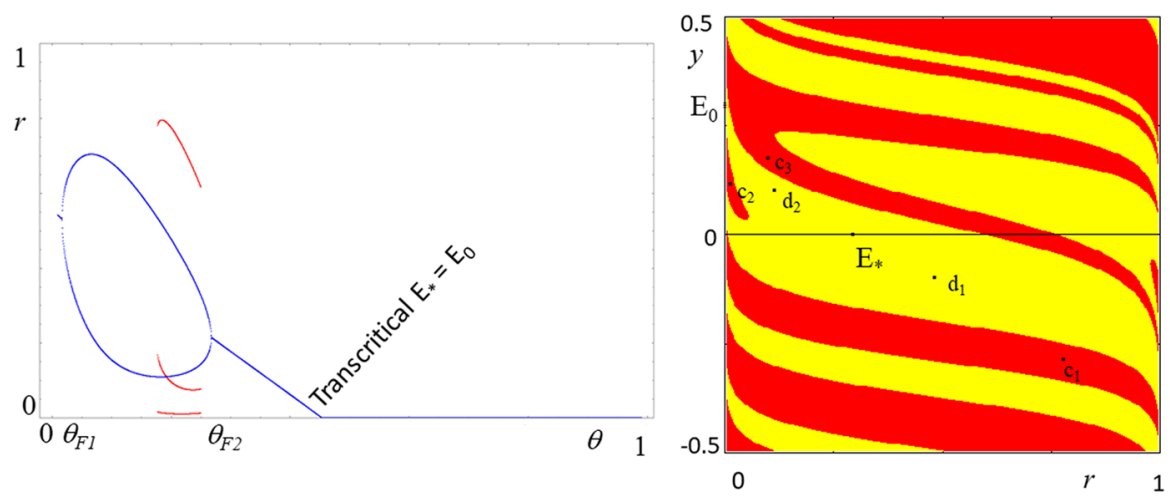

Fig. 2 Left: bifurcation diagram taking the cooperation coefficient $\theta \in[0,1]$ as bifurcation parameter, with the other parameters' values as in Fig. 1 (left) and $\omega=0.3$. Right: basins of attraction of the stable cycle of period 2 with periodic points $\left(d_{1}, d_{2}\right)$ around the equilibrium $E_{*}$ (yellow region) and of the coexisting stable cycle of period 3 with periodic points $\left(c_{1}, c_{2}, c_{3}\right)$ (red region) obtained for the same set of parameters as the bifurcation diagram in the left and with $\theta=0.2$ (color figure online)

the equilibrium $E_{*}$ exits the feasible region of the phase space after crossing over the boundary equilibrium $E_{0}$ through a transcritical (or stability exchange) bifurcation as discussed in Proposition 2. After this bifurcation, the only stable equilibrium is $E_{0}$ where only aggressive firms operate in the market. As already explained, this is due to the dominance of aggressive behavior that determines the extinction of cooperation as the game is played over and over. As cooperation is a dominated strategy for $\beta<0$, the presence of memory cannot change average fitness and, thus, cannot influence the stability properties of equilibrium $E_{0}$ but only the speed of convergence to $E_{0}$.

Another interesting situation is depicted in Fig. 3. Here, according to the seconddegree bifurcation condition (24), the stability of $E_{*}$ occurs when $\theta \in\left(\theta_{F_{1}}, \theta_{F_{2}}\right)$ where $\theta_{F_{1}} \approx-0.056$ and $\theta_{F_{2}} \approx 0.3277$. Being $\theta_{F_{1}}<0$, we observe instability of the equilibrium $E_{*}$ even for low values of the amount of cooperative behavior $\theta$. Instability of $E_{*}$ is here obtained by combining a high intensity of choice, that is, high agents' impatience, with low values of memory. The combined effect is that of bringing overshooting around the inner equilibrium $E_{*}$ for low levels of cooperation $\theta$. Overshooting reduces as $\theta$ is increased, and through this mechanism, the stability of $E_{*}$ is retrieved when $\theta \in\left(\theta_{F_{2}}, \widehat{\theta}\right)$, where $\widehat{\theta}$, as before, is the value of the transcritical bifurcation at which $E_{*}$ exits the unitary interval. Interestingly, when $E_{*}$ is feasible but unstable, the cycle of period 2 loses stability through period-doubling cascades of bifurcations and regains stability through period-halving cascades of bifurcations as $\theta$ is further increased in the interval of instability of $E_{*}$.

So, in economic models represented by nonlinear dynamical systems, two kinds of complexity can be evidenced, which are related to complex attracting sets and complex structure of the basins of attraction. The local stability analysis provides important directions to guide the study of the global properties of the dynamical system that are investigated numerically, such as the structure of the basins of attraction.

Another aspect that we want to briefly address here is related to the economic implications of the dynamics of the model. As we have already compared equilibrium 


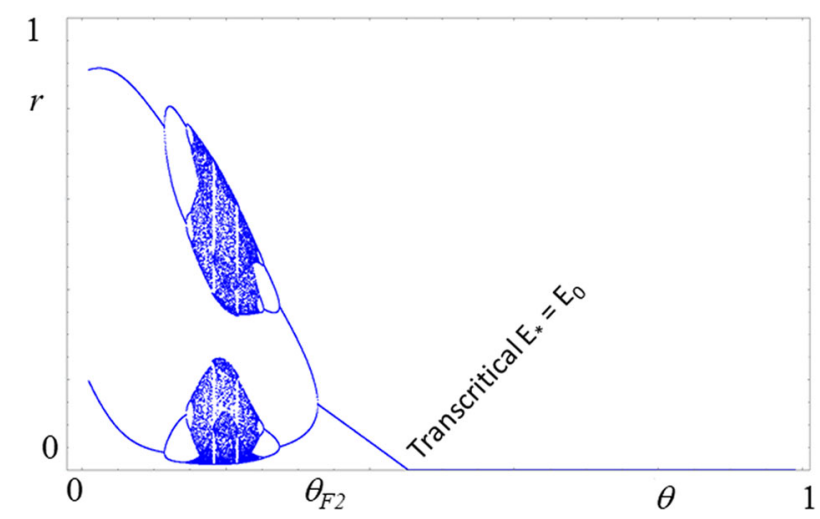

Fig. 3 Bifurcation diagram with $a=4, b=0.6, c=0.5, \omega=0.1, \rho=-0.7, \gamma=20$ and taking the cooperation coefficient $\theta \in[0,1]$ as bifurcation parameter

profits in the various cases, we here focus on total industry profits when dynamics fail to converge to equilibrium $E_{*}$.

Clearly, cooperative behavior leads here to the highest possible profit, whereas the minimum industry profit is reached when aggressive behavior from both players is always chosen. As previously seen, the evolutionary mechanism does not favor the prevalence of cooperation due to the immediate advantage of playing aggressively when the opponent plays cooperatively. So here we intend to analyze how the total profit of the industry varies when the fraction of cooperators does not converge to a boundary equilibrium but to a different attractor, for example to the internal equilibrium $E_{*}$ or to a periodic or chaotic attractor.

Figure 4, left panel, depicts for the same parameter setting as in Fig. 1, left panel, the total industry profit for varying levels of memory $\omega \in[0,0.5]$. The attractor here depicted shows total industry profits, defined as

$$
\Pi(t)=2 r(t)^{2} \pi_{s s}^{*}+r(t)(1-r(t))\left(\pi_{s v}^{*}+\pi_{v s}^{*}\right)+2(1-r(t))^{2} \pi_{v v}^{*}
$$

along the generic trajectory with initial condition $(r(0), y(0))=(0.5,-0.01)$ (blue points) and with initial condition $(r(0), y(0))=(0.65,-0.03)$ (red points). As we have seen before, in this example we have for some values of memory coexistence of attractors. In addition to the profit levels obtained under evolutionary dynamics, Fig. 4 also depicts the total industry profit that would occur without any type of evolutionary adjustment with all agents sticking to the same behavior. In particular, the horizontal dotted green, black and dotted black lines represent, respectively, total industry profits when both players always choose cooperative, Cournot-Nash and aggressive behavior, that is, $2 \pi_{s s}^{*}, \frac{2(a-c)^{2}}{9 b}, 2 \pi_{v v}^{*}$. We can easily see that as memory changes, the total profit of the companies is always greater than that obtained by always playing the aggressive strategy.

Furthermore, the average profit level on each chaotic attractor can differ quite substantially, as evident for the average profit along the chaotic attractor in blue (represented by the green curve) and in red (average profit represented by the gray curve). 

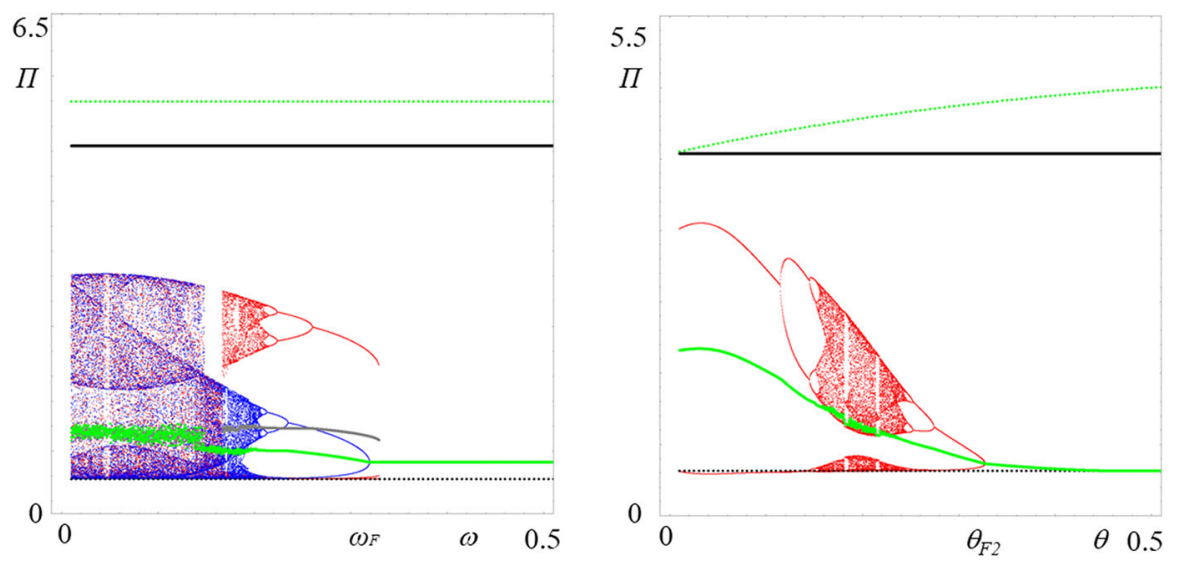

Fig. 4 Left: total industry profit for memory parameter $\omega \in[0,0.5]$ with the same parameters as in Fig. 1, left panel. The horizontal dotted green, black and dotted black lines represent, respectively, total industry profits when both players always choose cooperative, Cournot-Nash and aggressive behavior. Right: bifurcation diagram with parameters as in Fig. 3. The total industry profits are shown with $\theta$ ranging in the interval $[0,0.5]$

This shows that the initial conditions on the market, i.e., the initial share of cooperators and accumulated (past) profits, do matter for assessing the long-run average profits. We can also note an apparently paradoxical effect, related to the fact that by increasing the memory level $\omega$ of companies, the average total profit is decreasing precisely in the memory level.

A similar exercise is also proposed to highlight the effect of the level of cooperation $\theta$ on the total profit of the industry (25). For this reason, Fig. 4, right panel, proposes a bifurcation diagram with parameters as in Fig. 3 for total industry profits with $\theta$ ranging in the interval $[0,0.5]$. Clearly, the greater total profit here is no longer constant for the cooperative strategy (dotted green curve) since it grows monotonically with $\theta$ (even if the cooperative strategy is not chosen by agents because of its instability). Here, we note that along the two cycles existing before the chaotic attractor, there is the greatest oscillation of the total profit, even lower in a branch of cycle 2 than that obtained by playing the competitive strategy (dotted black horizontal line). Interestingly, we notice by inspecting Fig. 4 that the average profit (green curve) along the attractor is maximum for an intermediate level of cooperation $\theta$.

\section{Conclusions}

We have proposed an evolutionary oligopoly model in which agents can adopt cooperative or aggressive behavior. Cooperation should not be interpreted as collusion but as the intention of some companies to ease the level of competition to obtain more profits for themselves and the whole industry. Aggressive competition, on the other hand, intends to penalize rivals with the aim of weakening them. The various postulated behaviors influence the choices of the firms in terms of quantities they deliver to 
the market. The fitness of the various actions, however, is assessed on the basis of the profit obtained. Here, we consider not only the possibility that the most recent profit drives the fitness of each behavior but we construct a measure of the accumulated profit by introducing memory into the system, although memory weighs less on the previous behaviors through an exponential smoothing mechanism. As usual in evolutionary games, this fitness then steers the dynamic choice of agents' behaviors over time. Although it is possible to implement in various ways the quantities decisions by firms, here we focus on the simplest possible context, that of linear demand and linear costs and agents who choose based on Nash play, i.e., with firms offering quantities at Nash equilibrium for every possible game configuration.

We propose this specific example because it is possible to fully characterize the dynamic outcomes in terms of equilibria and their stability properties in the parameters space. The paper states the analytical conditions in the parameter space so that only a pure strategy dominates. In detail, in the linear case proposed in this paper, only aggressive behavior can be dominant. However, this strategy is the one that leads to the lowest level of profits in the industry.

In particular, when the amount of cooperative behavior is minimal and the amount of aggressive behavior is maximum $(\theta=0$ and $\rho=-1)$, this result reaffirms the well-known finding that the Walrasian equilibrium is evolutionarily stable for an evolutionary oligopoly while the Cournot-Nash equilibrium is not, see Vega-Redondo (1997), Radi (2017), Alós-Ferrer (2004), Apesteguia et al. (2010) and Schaffer (1989). Indeed, when the amount of cooperation is above the minimal level (i.e., $\theta>0$ ), our paper shows that the Walrasian equilibrium is evolutionarily robust not only with respect to the Cournot-Nash equilibrium but also with respect to the "collusive" equilibrium or any form of cooperation.

Furthermore, we show what happens when aggressive behavior is not a dominant strategy. In this case, an equilibrium with coexistence of the two behaviors exists. This equilibrium, however, can be destabilized if the agents have a high propensity to change strategies (intensity of choice) or, in some cases, if the memory level is not high enough. We briefly address the impact of instability of this coexistence equilibrium in terms of average industry profits, and we observe that the average industry profits can depend on the particular attractor of the system in cases of coexistence of attractors. Moreover, we detect cases in which there exists an intermediate level of memory that delivers the highest average profits along disequilibrium dynamics.

The dynamic analysis proposed in this paper gives the opportunity to learn a mathematical lesson as well, because in some ranges of the parameters such that the equilibrium is locally stable, coexisting periodic and chaotic attractors have been numerically observed, thus giving rise to strong path dependence of the dynamics. In fact, when the locally stable equilibrium coexists with a different kind of attractor, be it periodic or chaotic, each with its own basin of attraction, a typical situation of "corridor stability" occurs. In these cases, small perturbations (or shocks or historical accident) around the equilibrium are endogenously recovered by the dynamics of the system, whereas larger perturbations are amplified by the endogenous dynamics and lead to completely different (and nonstationary) disequilibrium dynamics. Thus, only an external control policy could force the system back to the original equilibrium. These dynamic scenarios clearly show the importance of a global analysis of nonlin- 
ear dynamical systems, which can often be performed only through heuristic methods obtained by a combination of analytical, geometrical and numerical tools. In fact, an analysis limited to a study of the local stability and bifurcations, based on the linear approximation of the model around the equilibrium points, sometimes may be quite incomplete and even misleading.

As stressed in Introduction and in the section dedicated to the model setup, the dynamic model proposed in this paper can be extended in several directions. Here, although we fully characterize the model in the parameters space, we assume that the levels of cooperative and/or aggressive behavior are fixed. The most relevant extension of this model that we leave open for future studies is related to endogenizing the levels of cooperation and/or aggression. Moreover, we would like to explore this model by considering nonlinear demand and/or cost functions or assuming different behavioral rules to decide next period production, such as best reply, gradient rules, LMA rule, etc. instead of Nash play assumed in this paper.

A further promising research direction consists of differentiating the interaction among firms, i.e., introducing a cooperation matrix instead of a simple cooperation coefficient. In other words, firm $i$ may be assumed to include firm $j$ profit inside its objective function according to the following network structure

$$
\max S=\pi_{i}+\sum_{j} \gamma_{i j} \pi_{j}
$$

where $\gamma_{i j} \in[-1,1]$ represent the entries of a connection matrix between the network of firms in the oligopoly market. We leave all these possible extensions to future works on the subject.

Acknowledgements We thank the participants to the 11th Nonlinear Economic Dynamics conference (NED-2019) in Kyiv, Davide Radi, the Editors and two anonymous reviewers for their constructive comments and insights. This work has been developed in the framework of the research project on "Models of behavioral economics for sustainable development" financed by DESP-University of Urbino, Italy. Fabio Lamantia acknowledges the support of the Czech Science Foundation (GACR) under project 20-16701S and VŠB-TU Ostrava under the SGS project SP2020/11.

Funding Open access funding provided by Università della Calabria within the CRUI-CARE Agreement. Università della Calabria and Czech Science Foundation GACR project 20-16701S.

\section{Compliance with ethical standards}

Conflict of interest The authors declare that there is no conflict of interest.

Open Access This article is licensed under a Creative Commons Attribution 4.0 International License, which permits use, sharing, adaptation, distribution and reproduction in any medium or format, as long as you give appropriate credit to the original author(s) and the source, provide a link to the Creative Commons licence, and indicate if changes were made. The images or other third party material in this article are included in the article's Creative Commons licence, unless indicated otherwise in a credit line to the material. If material is not included in the article's Creative Commons licence and your intended use is not permitted by statutory regulation or exceeds the permitted use, you will need to obtain permission directly from the copyright holder. To view a copy of this licence, visit http://creativecommons.org/licenses/by/4.0/. 


\section{Appendix}

Proof that $\pi_{s v}^{*}<\pi_{s s}^{*}<\pi_{v s}^{*}$

Consider first the inequality $\pi_{s v}^{*}<\pi_{s s}^{*}$, that is,

$$
\frac{(1-\theta \rho)(1-\theta)}{(3-\theta-\rho-\theta \rho)^{2}}<\frac{(1+\theta)}{(3+\theta)^{2}}
$$

which is equivalent to

$$
(1-\theta \rho)(1-\theta)(3+\theta)^{2}-(3-\theta-\rho-\theta \rho)^{2}(1+\theta)<0
$$

i.e.,

$$
(\theta+1)^{3} \rho-2\left(\theta^{2}+3\right)<0
$$

that is for $\rho<\frac{2\left(\theta^{2}+3\right)}{(\theta+1)^{3}}=g(\theta)$, which is trivially satisfied being $g(\theta)>0$ for all $\theta \geq 0$.

Now consider the inequality $\pi_{s s}^{*}<\pi_{v s}^{*}$, that is,

$$
\frac{(1+\theta)}{(3+\theta)^{2}}<\frac{(1-\theta \rho)(1-\rho)}{(3-\theta-\rho-\theta \rho)^{2}}
$$

which leads to

$$
\left(3 \theta^{2}+6 \theta-1\right) \rho+\theta^{2}-6 \theta-3<0
$$

that is $\rho<z(\theta)$ for $3 \theta^{2}+6 \theta-1 \geq 0$ and $\rho>z(\theta)$ when $3 \theta^{2}+6 \theta-1<0$ being $z(\theta)=\frac{-\theta^{2}+6 \theta+3}{3 \theta^{2}+6 \theta-1}$. As those conditions are always satisfied, inequality $\pi_{s s}^{*}<\pi_{v s}^{*}$ holds.

\section{Sign of $\alpha$ and $\beta$}

First, we show that $\alpha<0$. From the definition of $\alpha$ given in (17), with the expressions of the profits given in (8), (12), (13), (10), we get

$$
\begin{aligned}
\alpha= & \frac{(a-c)^{2}}{b}\left(\frac{\theta+1}{(3+\theta)^{2}}+\frac{\rho+1}{(3+\rho)^{2}}-\frac{(\theta-1)(\theta \rho-1)}{(3-\theta \rho-\theta-\rho)^{2}}\right. \\
& \left.-\frac{(\rho-1)(\theta \rho-1)}{(3-\theta \rho-\theta-\rho)^{2}}\right) \\
= & \frac{(a-c)^{2}}{b} \frac{-(\theta-\rho)^{2} H(\theta, \rho)}{(3+\theta)^{2}(3+\rho)^{2}(3-\theta \rho-\theta-\rho)^{2}}
\end{aligned}
$$


So, the negativity of $\alpha$ follows by showing that $H(\theta, \rho)>0$ where

$$
H(\theta, \rho)=\Psi \theta^{2}+\Lambda \theta+\Delta
$$

with

$$
\begin{aligned}
& \Psi=3 \rho^{2}+6 \rho-1<0, \text { for }-2.154 \approx \frac{-3-2 \sqrt{3}}{3}<\rho<\frac{-3+2 \sqrt{3}}{3} \approx 0.154 \\
& \Lambda=6 \rho^{2}-4 \rho-18<0, \text { for }-1.4305 \approx \frac{1-2 \sqrt{7}}{3}<\rho<\frac{1+2 \sqrt{7}}{3} \approx 2.097 \\
& \Delta=-\rho^{2}-18 \rho+27>0, \text { for }-19.39 \approx-9-6 \sqrt{3}<\rho<-9+6 \sqrt{3} \approx 1.39
\end{aligned}
$$

As we are considering $\rho \in[-1,0]$, we always have $\Psi<0, \Lambda<0$ and $\Delta>0$. By Descartes' rule of signs, equation $H(\theta, \rho)=0$ for a fixed $\rho \in[-1,0]$ has only one root $\theta^{*}>0$ such that $H\left(\theta^{*}, \rho\right)=0$. Moreover, for a fixed $\rho \in[-1,0], H(\theta, \rho)$ is a concave parabola in $\theta$, with $H(0, \rho)=\Delta>0$ and $H(1, \rho)=\Psi+\Lambda+\Delta=$ $8(1-\rho)^{2}>0$. Thus, the root $\theta^{*}$ is greater than one, and $H(\theta, \rho)>0$ for all $\rho \in[-1,0]$ and $\theta \in(0,1]$.

Now, let us consider the sign of $\beta=\pi_{s v}^{*}-\pi_{v v}^{*}$. From (17), with the expressions of the profits given in (12), (10) we obtain

$$
\beta=\frac{(a-c)^{2}}{b}\left(\frac{(\theta-1)(\theta \rho-1)}{(3-\theta \rho-\theta-\rho)^{2}}-\frac{\rho+1}{(\rho+3)^{2}}\right)
$$

Thus, $\beta>0$, that is, $\pi_{s v}^{*}>\pi_{v v}^{*}$, occurs whenever

$$
\frac{(\theta-1)(\theta \rho-1)}{(3-\theta \rho-\theta-\rho)^{2}}>\frac{\rho+1}{(\rho+3)^{2}}
$$

which is equivalent to

$$
0 \leq \theta<\frac{-\rho^{2}+6 \rho+3}{3 \rho^{2}+6 \rho-1}=h(\rho)
$$

By studying the strictly decreasing function $h(\rho)$ in the interval $[-1,0)$, it is then easy to show that it must be $-1 \leq \rho<3-2 \sqrt{3}$, so that $\beta>0$ provided that condition (20) holds.

\section{References}

Ahn TK, Ostrom E, Schmidt D, Shupp R, Walker J (2001) Cooperation in PD games: fear, greed, and history of play. Public Choice 106:137-155

Alger I, Weibull J (2013) HomoMoralis-preference evolution under incomplete information and assortative matching. Econometrica 81(6):2269-2302

Alós-Ferrer C (2004) Cournot versus Walras in dynamic oligopolies with memory. Int J Ind Organ 22(2):1217 
Andreoni J, Miller J (2002) Giving according to GARP: an experimental test of the consistency of preferences for altruism. Econometrica 70:737-753

Apesteguia J, Huck S, Oechssler J, Weidenholzer S (2010) Imitation and the evolution of Walrasian behavior: theoretically fragile but behaviorally robust. J Econ Theory 145(5):1603-1617

Bischi GI, Chiarella C, Kopel M, Szidarovszky F (2010) Nonlinear oligopolies: stability and bifurcations. Springer, New York

Bischi GI, Lamantia F (2002) Nonlinear duopoly games with positive cost externalities due to spillover effects. Chaos Solitons Fractals 13:805-822

Bischi GI, Kopel M (2003) Multistability and path dependence in a dynamic brand competition model. Chaos Solitons Fractals 18:561-576

Bischi GI, Lamantia F, Radi D (2015) An evolutionary Cournot model with limited market knowledge. J Econ Behav Organ 116:219-238

Bischi GI, Lamantia F, Scardamaglia B (2020). On the influence of memory on complex dynamics of evolutionary oligopoly models. Nonlinear Dyn. https://doi.org/10.1007/s11071-020-05827-9

Bischi GI, Merlone U, Pruscini E (2018) Evolutionary dynamics in club goods binary games. J Econ Dyn Control 91:104-119

Cabrales A, Sobel J (1992) On the limit points of discrete selection dynamics. J Econ Theory 57:407-419

Cerboni-Baiardi L, Naimzada AK (2018) An evolutionary model with best response and imitative rules. Decis Econ Finance 41(2):313-333

Charness G, Rabin M (2002) Understanding social preferences with simple tests. Quart J Econ 117(3):817869

Cyert RM, DeGroot MH (1973) An analysis of cooperation and learning in a duopoly context. Am Econ Rev 63(1):24-37

D'Aspremont C, Jacquemin A (1988) Cooperative and noncooperative R\&D in duopoly with spillovers. Am Econ Rev 78(5):1133-1137

De Giovanni D, Lamantia F (2016) Control delegation, information and beliefs in evolutionary oligopolies. J Evol Econ 26-5:1089-1116

Dohtani A, Inaba T, Osaka H (2007) Corridor stability of the neoclassical steady state. In: Asada T, Ishikawa $\mathrm{T}$ (eds) Time and space in economics. Springer, Tokyo, pp 129-143

Elaydi SN (1995) An introduction to difference equations. Springer, New York

Fershtman C, Judd KL (1987) Equilibrium incentives in oligopoly. Am Econ Rev 77(5):926-940

Gandolfo G (2010) Economic dynamics, 4th edn. Springer, Berlin

Hofbauer J, Sigmund K (1998) Evolutionary games and population dynamics. Cambridge University Press, Cambridge

Hofbauer J, Sigmund K (2003) Evolutionary game dynamics. Bull Am Math Soc 40(4):479-519

Hommes CH, Kiseleva T, Kuznetsov Y, Verbic M (2012) Is more memory in evolutionary selection (de)stabilizing? Macroecon Dyn 16:335-357

Hommes CH, Ochea MI, Tuinstra J (2018) Evolutionary competition between adjustment processes in cournot oligopoly: instability and complex dynamics. Dyn Games Appl 8:822-843

Janssen MA (2008) Evolution of cooperation in a one-shot Prisoner's Dilemma based on recognition of trustworthy and untrustworthy agents. J Econ Behav Organ 65(3-4):458-471

Königstein M, Müller W (2000) Combining rational choice and evolutionary dynamics: the indirect evolutionary approach. Metroeconomica 51(3):235-256

Kopel M, Lamantia F, Szidarovszky F (2014) Evolutionary competition in a mixed market with socially concerned firms. J Econ Dyn Control 48:394-409

Kopel M, Lamantia F (2018) The persistence of social strategies under increasing competitive pressure. J Econ Dyn Control 91:71-83

Leijonhufvud A (1973) Effective demand failures. Swed J Econ 75(1):27-48

Leimar O, Hammerstein P (2001) Evolution of cooperation through indirect reciprocity. Proc R Soc B 268:745-753

Medio A, Lines M (2001) Nonlinear dynamics. Cambridge University Press, Cambridge

Nowak M, Sigmund K (2005) Evolution of indirect reciprocity. Nature 437:1291-1298

Puu T (1991) Chaos in duopoly pricing. Chaos Solitons Fractals 1(6):573-581

Radi D (2017) Walrasian versus Cournot behavior in an oligopoly of boundedly rational firms. J Evol Econ 27:933-961

Schaffer AE (1989) Are profit-maximisers the best survivors? A Darwinian model of economic natural selection. J Econ Behav Organ 12(1):29-45 
Smith JM (1982) Evolution and the theory of games. Cambridge University Press, Cambridge

Vallée T, Yildizoglu M (2009) Convergence in the finite Cournot oligopoly with social and individual learning. J Econ Behav Organ 72:670-690

Vega-Redondo F (1997) The evolution of Walrasian behavior. Econometrica 65(2):375-384

Vriend NJ (2000) An illustration of the essential difference between individual and social learning, and its consequences for computational analyses. J Econ Dyn Control 24:1-19

Publisher's Note Springer Nature remains neutral with regard to jurisdictional claims in published maps and institutional affiliations. 